\title{
Effect of a methionine-supplemented diet on the blood pressure of Sprague-Dawley and deoxycorticosterone acetate-salt hypertensive rats
}

\author{
Sophie Robin ${ }^{1 \star}$, Véronique Maupoil ${ }^{1}$, Pascal Laurant ${ }^{1}$, Alain Jacqueson ${ }^{2}$ and Alain Berthelot ${ }^{1}$ \\ ${ }^{1}$ Laboratoire de Physiologie, Pharmacologie et Nutrition Préventive Expérimentale and \\ ${ }^{2}$ Laboratoire de Toxicologie, UFR Médecine et Pharmacie, 4 Place Saint-Jacques, 25030 Besançon cedex, France
}

(Received 12 September 2003 - Revised 1 February 2004 - Accepted 6 February 2004)

\begin{abstract}
The objectives of the present study were to evaluate the effects of a methionine-supplemented diet on systolic blood pressure (BP) and vasomotor functions in Sprague-Dawley (SD) and deoxycorticosterone acetate (DOCA)-salt hypertensive rats. SD and DOCA rats were fed a normal or a methionine $(8 \mathrm{~g} / \mathrm{kg})$-supplemented diet for 10 weeks. Systolic BP was monitored and plasma homocysteine, methionine and cysteine levels were determined at the end of the experiment. Vasoconstriction and vasodilatation of aortic rings were measured. The methionine-supplemented diet induced a greater increase in homocysteinaemia concentration in DOCA rats than in SD rats and an increase in plasma cysteine concentration in DOCA rats. This diet was associated with an increase in systolic BP in SD rats and with a lesser development of DOCA-salt hypertension. An enhanced aortic constriction and a decreased responsiveness to acetylcholine, bradykinin and sodium nitroprusside in the SD rats fed the methionine-rich diet were consistent with the elevated systolic BP. In DOCA rats the increased responsiveness to bradykinin was in accordance with the systolic BP-lowering effect. In conclusion, the methionine-enriched diet cannot simply be considered as model of hyperhomocysteinaemia, since other metabolites and mechanisms seemed to be implicated in these complex interactions. The differential vasopressive effect of the methionine supplementation in SD and DOCA rats, and in particular the lowering of systolic BP obtained with a greater degree of hyperhomocysteinaemia in DOCA rats, suggest that more complex interactions exist between hyperhomocysteinaemia and BP than the simple positive association described previously.
\end{abstract}

Deoxycorticosterone acetate-salt hypertensive rats: Homocysteine: Aortic rings

An association between elevated plasma homocysteine (Hcy) concentrations and peripheral arterial disease has been shown by several epidemiological studies (Taylor et al. 1999; Mansoor et al. 2000). Experimental and clinical studies have demonstrated that hyperhomocysteinaemia (HHcy) is associated with alterations in endothelial morphology (McCully 1969; Matthias et al. 1996), endothelium-dependent vasodilatation (Kanani et al. 1999; Ungvari et al. 1999; Hanratty et al. 2001), smooth muscle cell proliferation, collagen synthesis (Majors et al. 1997) and deterioration of elastic material of the vascular wall (Charpiot et al. 1998).

Given the impairment of vasoregulatory mechanisms, vascular wall structure and properties, one would predict a positive association between HHcy and blood pressure (BP). However, the link between HHcy and hypertension is controversial. In human subjects, elevated Hcy levels were either strongly and independently associated with isolated systolic hypertension (Sutton-Tyrrell et al. 1997) or associated with higher diastolic and mean arterial BP
(Fiorina et al. 1998). Conversely, it has been reported that subjects with a common mutation of the methylenetetrahydrofolate reductase gene, which may account for elevated plasma Hcy concentrations, had a significantly lower BP than subjects with other genotypes in the general population (Nakata et al. 1998). In animals, 4 months of a methionine (Met)-enriched diet was reported to induce a mild HHcy and both systolic and diastolic hypertension in normotensive minipigs (Rolland et al. 1995), whereas an absence of variation in BP has been reported for normotensive rats fed a Met-enriched diet (Matthias et al. 1996; Ungvari et al. 1999). Recently, we showed that a Met-enriched diet increased the systolic BP in normotensive Wistar-Kyoto rats and decreased it in spontaneously hypertensive rats (SHR), while inducing a 3-fold increase of plasma Hcy in both strains (Robin et al. 2003). An attenuation of the development of hypertension was reported in female SHR, whereas an accelerating effect was observed in males after Met overloading, which induced a similar 3-fold increase in serum Hcy in each gender (Yen \& Lau, 2002). It is

\footnotetext{
Abbreviations: BP, blood pressure; DOCA, deoxycorticosterone acetate; EC 50 , half effective concentration; Hcy, homocysteine; HHcy, hyperhomocysteinaemia; Met, methionine; SD, Sprague-Dawley; SHR, spontaneously hypertensive rat. * Corresponding author: Dr Sophie Robin, fax +33 3816656 91, email ROBINRobinsophie@aol.com.
} 
therefore clear that the interaction between HHcy and BP is complex and that hypertension and/or gender are important factors to consider.

The difficulty in studying a disease process such as hypertension begins with the fundamental assertion that the aetiology of hypertension is heterogeneous. The most frequently encountered human type of hypertension is primary (also often called essential) hypertension, which is not purely represented by genetic animal models (e.g. SHR, Dahl, transgenic). Secondary hypertension is often represented by the mineralocorticoid-salt model, which resembles the clinical situation of aldosterone excess and by Goldblatt hypertension induced by constriction of a renal artery. Our previous study performed in SHR led us to extend our studies to a model of secondary hypertension. Since aldosterone was known to increase the expression and activity of $S$-adenosylhomocysteine hydrolase, which catabolizes $S$-adenosylhomocysteine in Hcy (Stockand et al. 2001), we used the mineralocorticoidsalt model of hypertension in order to induce a greater degree of HHcy than in the SHR model. In addition, this mineralocorticoid-salt model represents a low-renin form of hypertension with salt and water retention as against the high-renin SHR model (Pinto et al. 1997).

The present study reports the effect of a Met-enriched diet upon the development of deoxycorticosterone acetate (DOCA)-salt hypertension in male Sprague-Dawley (SD) rats. Since vascular and thiol metabolite alterations have been observed in Wistar-Kyoto rats and SHR (Robin et al. 2003), the vasomotor function of aortic rings, the plasma levels of Hcy and its metabolic derivatives Met, cysteine and GSH, were also investigated.

\section{Methods}

\section{Chemicals and reagents}

L-Noradrenaline hydrochloride, acetylcholine chloride, sodium nitroprusside, bradykinin, calcium chloride, DL-Met, DL-homocysteine, tri- $n$-butylphosphine and 7fluoro-2,1,3-benzoxadiazole-4-sulfonamide were purchased from Sigma (La Verpillère, France). Other reagents were of analytical grade.

\section{Animals}

SD male rats weighing $80-100 \mathrm{~g}$ were purchased from IFFA CREDO, L'Arbesle, France. Male rats were used to avoid a gender-dependent response (Yen \& Lau, 2002). The rats were housed in polyethylene cages in an environment with a controlled temperature of $22 \pm 2^{\circ} \mathrm{C}$, constant humidity (50-60\%) and a $12 \mathrm{~h}$ light-dark cycle. They had free access to food and water.

\section{Experimental procedures}

Animals were randomized into two groups (twenty-four per group). One group (DOCA group) was made hypertensive by thoracic subcutaneous implantation of four pellets of $25 \mathrm{mg}$ DOCA $(100 \mathrm{mg})$ and by addition of $\mathrm{NaCl}(0.9 \mathrm{~g} / \mathrm{l})$ in drinking water. The other group (SD group) was sham-operated and received distilled water as drinking water. The same day, each group was randomized into two subgroups. SD and DOCA control groups were fed for 10 weeks on a normal semisynthetic diet (per $\mathrm{kg}$ diet): casein $200 \mathrm{~g}$, cellulose $60 \mathrm{~g}$, maize starch $400 \mathrm{~g}$, granulated sugar $210 \mathrm{~g}$, maize oil $25 \mathrm{ml}$, peanut oil $25 \mathrm{ml}$, vitamin mix $10 \mathrm{~g}$, mineral mix $70 \mathrm{~g}$ (Robin et al. 2003). The diet contained (per kg): Met $6.50 \mathrm{~g}$, pyridoxine $10 \mathrm{mg}$, folic acid $0.05 \mathrm{mg}$, cyanocobalamin $0.05 \mathrm{mg}$. The experimental groups SD-Met and DOCA-Met were fed for the same period with the semisynthetic diet that was supplemented with an additional $8 \mathrm{~g}$ DL$\mathrm{Met} / \mathrm{kg}$, giving a total of $14.50 \mathrm{~g}$ Met $/ \mathrm{kg}$ diet.

Systolic BP was measured in unanaesthetized restrained rats by the indirect tail-cuff method using a sphygmomanometer (PE-3000; Narco-Biosystems, Houston, TX, USA) as previously described (Robin et al. 2003). The systolic $\mathrm{BP}$ was measured $0,1,3,5,7$ and 10 weeks after the beginning of the experiment.

On day 40 of the experimental period, each rat was housed alone in a polyethylene cage and for $2 \mathrm{~d}$ the quantity of food and drink consumed and urine produced was measured. Urine $(5 \mathrm{ml})$ from each rat was stored at $-20^{\circ} \mathrm{C}$ in polyethylene tubes until analysis.

At the end of the experiment, the rats were anaesthetized with sodium pentobarbital $(60 \mathrm{mg} / \mathrm{kg}$ body weight, intraperitoneally). Abdominal aorta blood was collected and placed in EDTA-vacutainer and sodium heparinate-vacutainer tubes, immediately cooled on iced water and centrifuged at $4000 \mathrm{rpm}$ for $10 \mathrm{~min}$ at $4^{\circ} \mathrm{C}$. Portions of plasma were stored at $-20^{\circ} \mathrm{C}$ until analysis. The thoracic aorta was dissected for the study of vascular reactivity.

\section{Ex vivo isolated aorta preparation}

Isolated aorta preparations were performed as previously described (Robin et al. 2003) using a physiological salt solution: $118 \mathrm{~mm}-\mathrm{NaCl}, \quad 1.2 \mathrm{mM}-\mathrm{KH}_{2} \mathrm{PO}_{4}, \quad 2.5 \mathrm{mM}-\mathrm{CaCl}_{2}$, $4.7 \mathrm{~mm}-\mathrm{KCl}, 1.2 \mathrm{mM}-\mathrm{MgSO}_{4}, 25 \mathrm{mM}-\mathrm{NaHCO}_{3}, 12 \mathrm{mM}-\mathrm{glu}-$ cose (pH 7.4) kept at $37^{\circ} \mathrm{C}$ and constantly bubbled with $\mathrm{O}_{2}-\mathrm{CO}_{2}(95: 5, \mathrm{v} / \mathrm{v})$.

In the first series of experiments, which were performed on aortic rings with endothelium, a cumulative concentration-response curve to noradrenaline $\left(10^{-10}-10^{-5} \mathrm{M}\right)$ was obtained. Then, with rings precontracted with a concentration of noradrenaline inducing $70 \%$ maximal contraction, a cumulative concentration-response curve to acetylcholine $\left(10^{-9}-10^{-4} \mathrm{M}\right)$ or bradykinin $\left(10^{-10}\right.$ $\left.10^{-5} \mathrm{M}\right)$ was recorded.

In experiments that were performed with rings without endothelium, a cumulative concentration-response curve to sodium nitroprusside $\left(10^{-11}-10^{-6} \mathrm{M}\right)$ and a cumulative concentration-response curve to $\mathrm{CaCl}_{2}\left(10^{-6}-10^{-2} \mathrm{M}\right)$ after a re-equilibration in $\mathrm{Ca}$-free $\mathrm{K}$ depolarizing Krebs solution $(100 \mathrm{~mm}-\mathrm{KCl}, 18 \mathrm{mM}-\mathrm{NaCl}$ and without added $\mathrm{CaCl}_{2}$ ) were obtained.

\section{Biochemical analysis}

Determination of plasma methionine, homocysteine, cysteine and GSH. As previously described (Robin et al. 2003), total Hcy, cysteine and GSH concentrations in plasma were 
measured by HPLC with fluorimetric determination after derivation of thiols with 7-fluoro-2,1,3-benzoxadiazole-4sulfonamide. Total Met concentration was measured by HPLC with electrochemical detection.

Determination of sodium content in plasma and urine. $\mathrm{Na}$ in plasma and urine was analysed by electrolyte analyser AVL-988 (AVL Instruments Médicaux, Cergy Pontoise, France) using a Na-selective electrode after appropriate dilution in distilled water.

\section{Statistical analyses}

Results were expressed as mean values with their standard errors. For each variable, statistical analysis was performed with two-way ANOVA, the two factors being rat strain and treatment, followed by inter-group pair-wise comparisons with Tukey's post hoc test when significant $(P<0.05)$ differences were detected.

\section{Results}

\section{Metabolism and plasma and urine sodium levels}

As expected, the DOCA rats had a greater fluid intake, urine volume and urine $\mathrm{Na}$ excretion. The plasma $\mathrm{Na}$ concentration also increased in the DOCA control group and this value tended to normalize in the DOCA-Met group (Table 1).
There was a tendency for Met-fed rats to have a higher food and fluid intake and urine volume compared with control diet-fed rats, though this did not achieve statistical significance. The daily Met intake was similar in SD and DOCA rats and the extra Met intake was about $680 \mathrm{mg}$ / $\mathrm{kg}$ body weight in the Met-supplemented rats (Table 1).

\section{Changes in body weight and organ weights}

The change in the body weight of rats over the 10 weeks of the experiment was similar in the four experimental groups, from about $130 \mathrm{~g}$ at the beginning of the experiment to about $430 \mathrm{~g}$ at week 10 .

The kidneys and hearts of the DOCA rats were significantly $(P<0 \cdot 01)$ heavier than those of SD rats. There was no significant difference between the weights of the livers of SD and DOCA groups.

The Met-enriched diet had no significant effect upon either total body or isolated organ weight (Table 2).

\section{Total plasma homocysteine, methionine, cysteine and GSH levels}

After 10 weeks of a Met-supplemented diet, plasma Hcy concentrations were $2 \cdot 7$-fold $(P<0 \cdot 01)$ greater in SD rats and $7 \cdot 1$-fold $(P<0 \cdot 01)$ higher in DOCA rats than baseline values; thus, the plasma Hcy level was significantly

Table 1. Daily methionine intake, food and fluid intake, urine volume and plasma and urine sodium in control and methionine-supplemented Sprague-Dawley and deoxycorticosterone-salt hypertensive rats§ (Mean values with their standard errors for twelve rats per group)

\begin{tabular}{|c|c|c|c|c|c|c|c|c|}
\hline & \multicolumn{4}{|c|}{ SD } & \multicolumn{4}{|c|}{ DOCA } \\
\hline & \multicolumn{2}{|c|}{ Control } & \multicolumn{2}{|c|}{ Met } & \multicolumn{2}{|c|}{ Control } & \multicolumn{2}{|c|}{ Met } \\
\hline & Mean & SEM & Mean & SEM & Mean & SEM & Mean & SEM \\
\hline Food intake (g/kg per d) & $63 \cdot 3$ & $2 \cdot 6$ & $74 \cdot 2$ & $3 \cdot 3$ & 63.5 & $3 \cdot 3$ & $76 \cdot 6$ & $8 \cdot 1$ \\
\hline Met intake (mg/kg per d) & 411.5 & $16 \cdot 9$ & $1075 \cdot 9^{*}$ & $47 \cdot 85$ & $412 \cdot 8$ & 21.5 & $1110 \cdot 7 \dagger$ & $24 \cdot 7$ \\
\hline Fluid intake (ml/kg per $\mathrm{d}$ ) & $72 \cdot 3$ & $6 \cdot 2$ & 90.9 & $8 \cdot 2$ & $334 \cdot 9^{*}$ & $42 \cdot 1$ & $378 \cdot 2 \ddagger$ & $49 \cdot 1$ \\
\hline Urine volume $(\mathrm{ml} / \mathrm{kg}$ per $\mathrm{d})$ & $41 \cdot 2$ & 4.6 & $54 \cdot 2$ & $6 \cdot 0$ & $313 \cdot 4^{\star}$ & $40 \cdot 3$ & $361.5 \ddagger$ & $41 \cdot 8$ \\
\hline Plasma $\mathrm{Na}(\mathrm{mm})$ & $140 \cdot 1$ & 0.37 & $140 \cdot 1$ & 0.70 & $142 \cdot 7^{\star}$ & 0.60 & $141 \cdot 2$ & 0.43 \\
\hline Urine $\mathrm{Na}(\mathrm{mmol} / \mathrm{kg}$ per $\mathrm{d})$ & $4 \cdot 9$ & 0.3 & $6 \cdot 1$ & 0.4 & $24 \cdot 0^{*}$ & $5 \cdot 2$ & $22 \cdot 3 \ddagger$ & $6 \cdot 7$ \\
\hline
\end{tabular}

SD, Sprague-Dawley rats; DOCA, deoxycorticosterone-salt hypertensive rats; Met, methionine.

Mean values were significantly different from that of the SD control group: ${ }^{\star} P<0.01$.

Mean value was significantly different from that of the DOCA control group: $\dagger P<0.01$.

Mean values were significantly different from those of the SD Met group: $\ddagger P<0.01$.

$\S$ For details of diets and procedures, see p. 858.

Table 2. Organ weights in control and methionine-supplemented Sprague-Dawley and deoxycorticosteronesalt hypertensive rats after 10 weeks of treatment

(Mean values with their standard errors for twelve rats per group)

\begin{tabular}{|c|c|c|c|c|c|c|c|c|}
\hline & \multicolumn{4}{|c|}{ SD } & \multicolumn{4}{|c|}{ DOCA } \\
\hline & \multicolumn{2}{|c|}{ Control } & \multicolumn{2}{|c|}{ Met } & \multicolumn{2}{|c|}{ Control } & \multicolumn{2}{|c|}{ Met } \\
\hline & Mean & SEM & Mean & SEM & Mean & SEM & Mean & SEM \\
\hline Kidney (g) & 1.34 & 0.04 & 1.40 & 0.04 & $2.43^{*}$ & 0.17 & $2.45 \dagger$ & 0.18 \\
\hline Liver (g) & $14 \cdot 11$ & 0.50 & 14.05 & 0.62 & $14 \cdot 19$ & 0.50 & $14 \cdot 46$ & 0.45 \\
\hline Heart (g) & $1 \cdot 13$ & 0.02 & $1 \cdot 10$ & 0.03 & $1.57^{\star}$ & 0.04 & $1.53 \dagger$ & 0.05 \\
\hline
\end{tabular}

SD, Sprague-Dawley rats; DOCA, deoxycorticosterone-salt hypertensive rats; Met, methionine.

Mean values were significantly different from those of the SD control group: ${ }^{*} P<0.01$.

Mean values were significantly different from those of the SD Met group: $† P<0.01$.

‡For details of diets and procedures, see p. 858 
$(P<0.01)$ increased in the DOCA-Met rats (21 (SEM 6) $\mu \mathrm{M})$ compared with the SD-Met rats (11 (SEM 2) $\mu \mathrm{M})$.

The plasma Met level was also significantly higher in SD $(P<0.05)$ and DOCA $(P<0.01)$ rats on the high-Met diet. The Met-supplemented diet also induced an increase in plasma cysteine concentration in DOCA-Met rats compared with SD-Met rats $(P<0 \cdot 05)$, where it had no effect. Plasma GSH levels were similar in all groups of animals (Fig. 1).

(A)

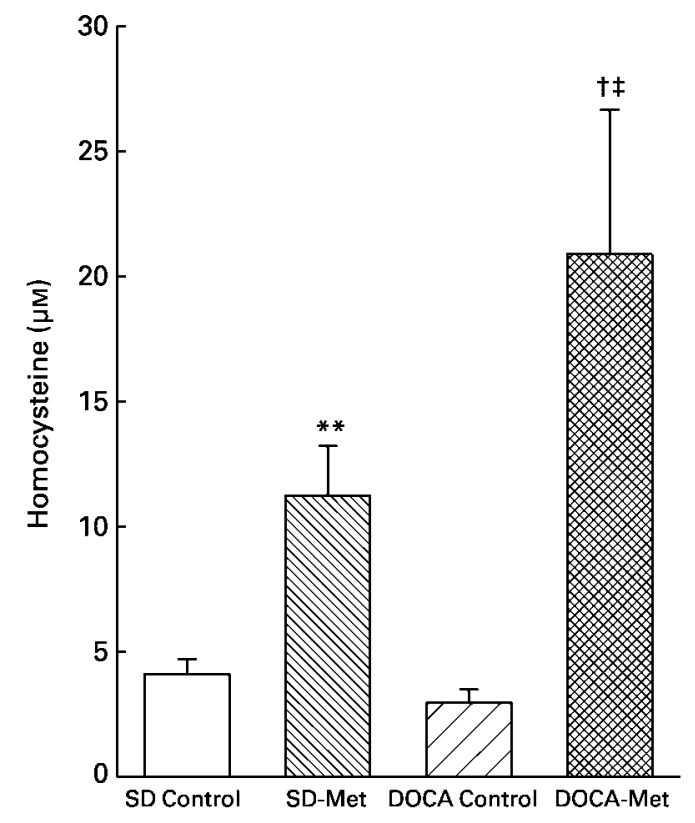

(C)

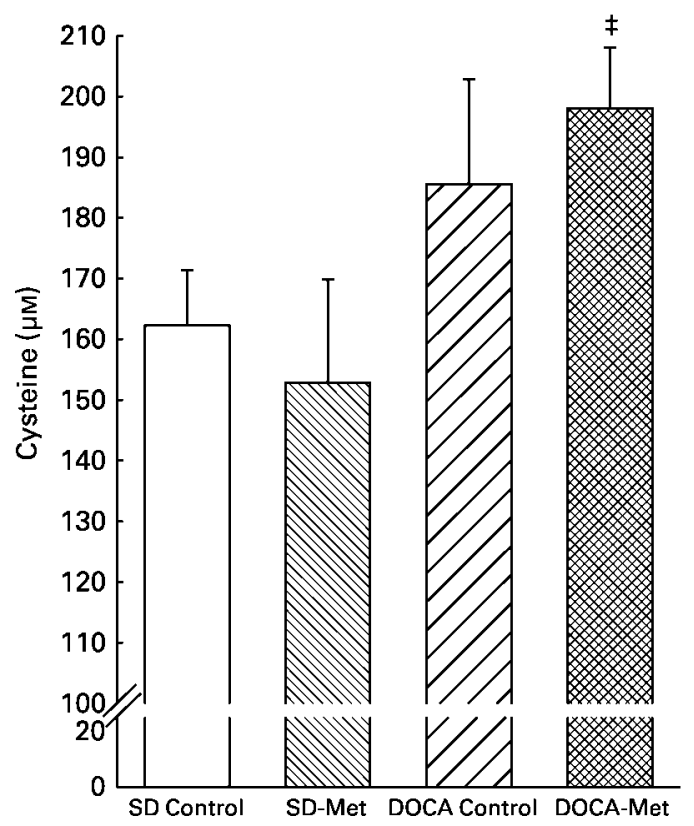

Effect of methionine-supplemented diet on systolic blood pressure

At the beginning of the experiment, the systolic BP of SD and DOCA rats was similar at about $105-110 \mathrm{mmHg}$. By week 5 of the experiment, the systolic BP of DOCA control rats was considerably elevated $(P<0 \cdot 01)$ compared with that of SD control rats. Starting from week 5 , the Met-supplemented diet significantly decreased the systolic

(B)

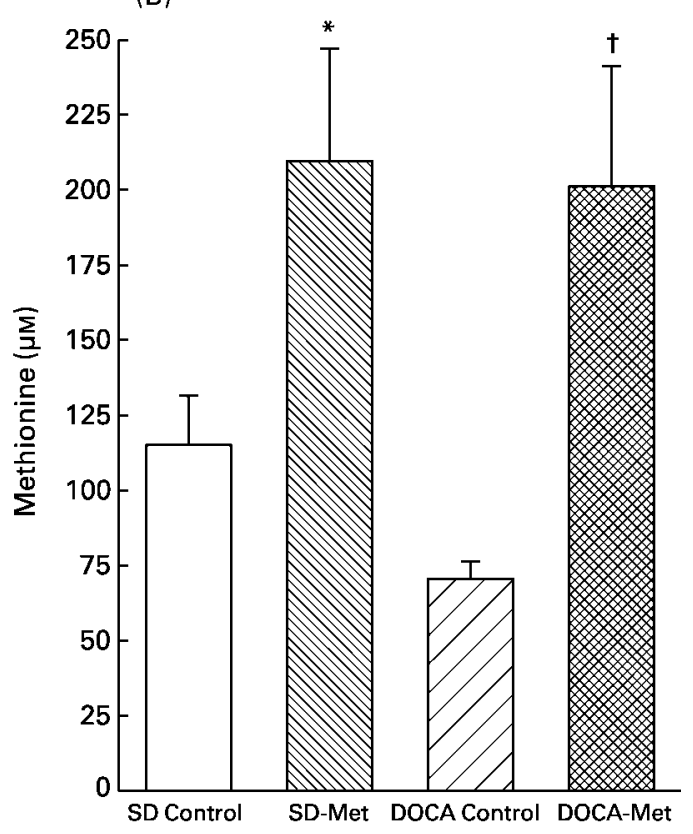

(D)

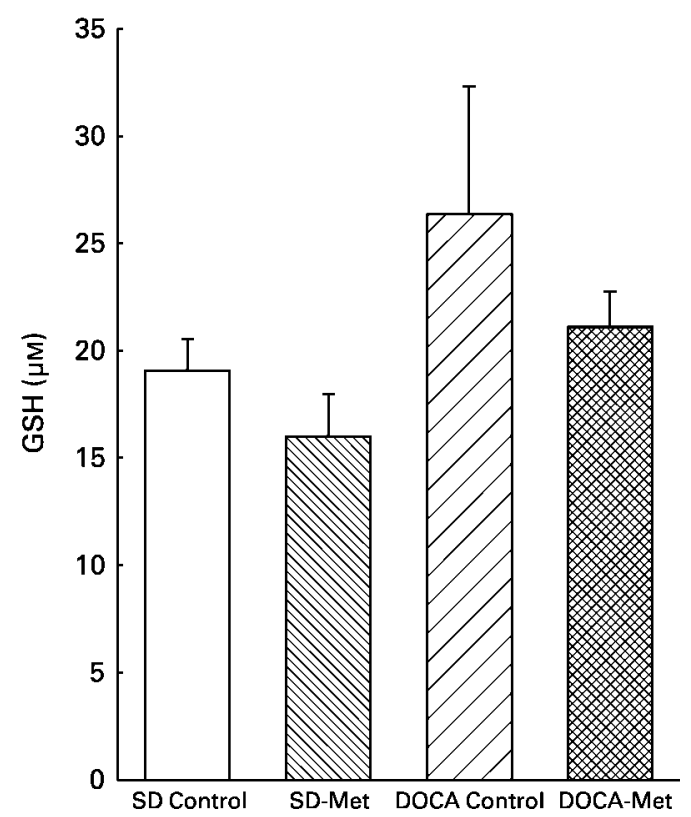

Fig. 1. Effect of a control or methionine (Met)-supplemented diet on plasma homocysteine (A), Met (B), cysteine (C) and GSH (D) in Sprague-Dawley (SD) and deoxycorticosterone acetate (DOCA)-salt hypertensive rats. For details of diets and procedures, see p. 858. Values are means with their standard errors shown by vertical bars for twelve rats per group. Mean values for SD-Met group were significantly different from those of the SD control group: ${ }^{\star} P<0.05,{ }^{\star *} P<0.01$. Mean value for DOCA-Met group was significantly different from that of the DOCA control group: $\dagger P<0.01$. Mean values for DOCA Met group were significantly different from that of the SD-Met group: $\ddagger P<0.05$. 


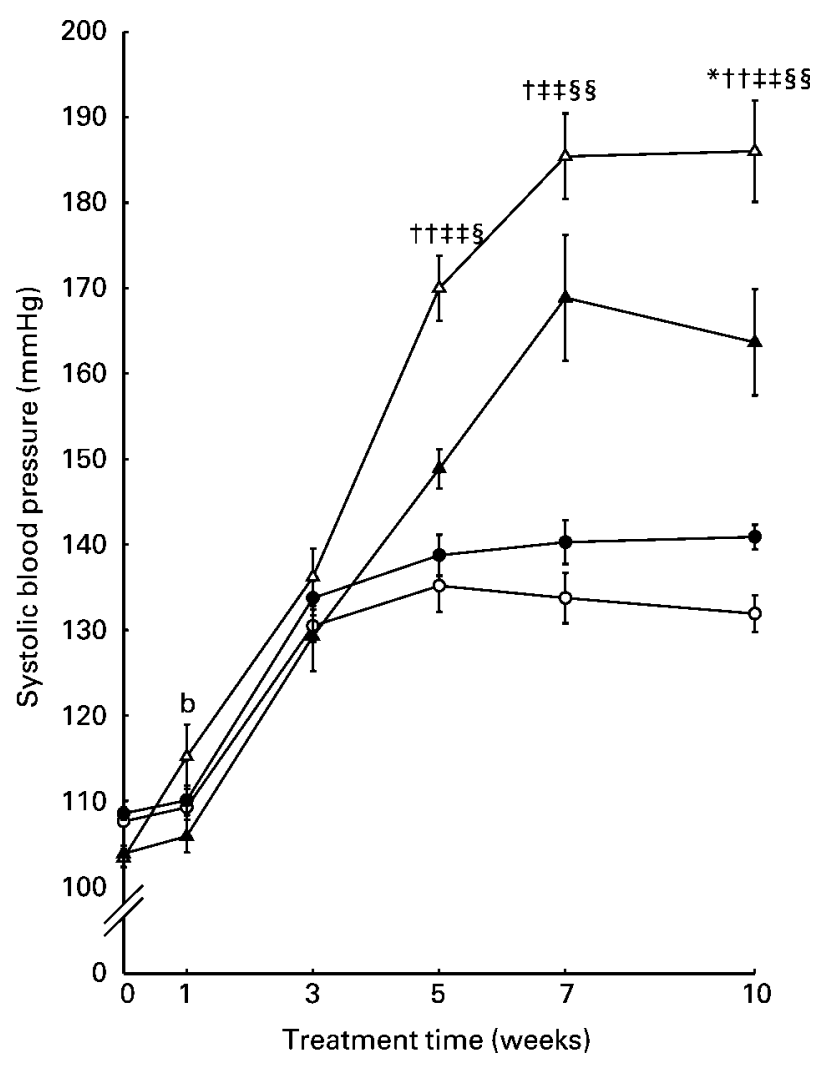

Fig. 2. Effect of a control or methionine (Met)-supplemented diet on systolic blood pressure in Sprague-Dawley (SD) and deoxycorticosterone acetate (DOCA)-salt hypertensive rats. $\mathrm{O}, \mathrm{SD}$ control -, SD-Met; $\triangle$, DOCA control; $\boldsymbol{\Lambda}$, DOCA-Met. For details of diets and procedures, see p. 858 . Values are means with their standard errors shown by vertical bars for twelve rats per group. Mean value for SD-Met group was significantly different from that of the SD control group: ${ }^{*} P<0 \cdot 05$. Mean values for DOCA-Met group were significantly different from those of the DOCA control group: $\dagger P<0.05$, $\dagger+P<0.01$. Mean values for DOCA control group were significantly different from those of the SD control group: $\ddagger \ddagger P<0.01$. Mean values for DOCA-Met group were significantly different from those of the SD-Met group: $\S P<0.05, \S \S P<0.01$.

BP of DOCA rats (about $-20 \mathrm{mmHg}, P<0 \cdot 01$ ). By week 7 , the Met-supplemented diet significantly $(P<0.05)$ increased the systolic BP of SD rats. Thus, the Met-supplemented diet induced a lesser development of hypertension in DOCA rats while a slight increase of the systolic BP was observed in the SD rats (Fig. 2).

\section{Aortic reactivity to noradrenaline and calcium chloride}

Noradrenaline evoked a more effective constriction of aortic rings from control DOCA rats than SD control rats, whereas the maximum contraction was not modified. The response to noradrenaline was improved in the DOCA-Met group (half effective concentration $\left(\mathrm{EC}_{50}\right)$ $0.48 \times 10^{-8}$ (SEM $0.20 \times 10^{-8}$ ) M, maximum 111.1 (SEM $5.1) \%$ ) compared with the DOCA control group $\left(\mathrm{EC}_{50}\right.$ $1.35 \times 10^{-8}\left(\right.$ SEM $\left.0.50 \times 10^{-8}\right) \mathrm{M}$, maximum 97.5 (SEM 4.2) $\% ; P<0.01)$. The Met-enriched diet induced a slight increase in the sensitivity to noradrenaline in the normotensive SD rats $\left(\mathrm{EC}_{50} 2.21 \times 10^{-8}\left(\right.\right.$ SEM $\left.0.36 \times 10^{-8}\right) \mathrm{M}$ in the SD group v. $4.27 \times 10^{-8}\left(\right.$ SEM $\left.0.57 \times 10^{-8}\right) \mathrm{M}$ in the SD-Met group; $P<0.05$ ) (Fig. 3).

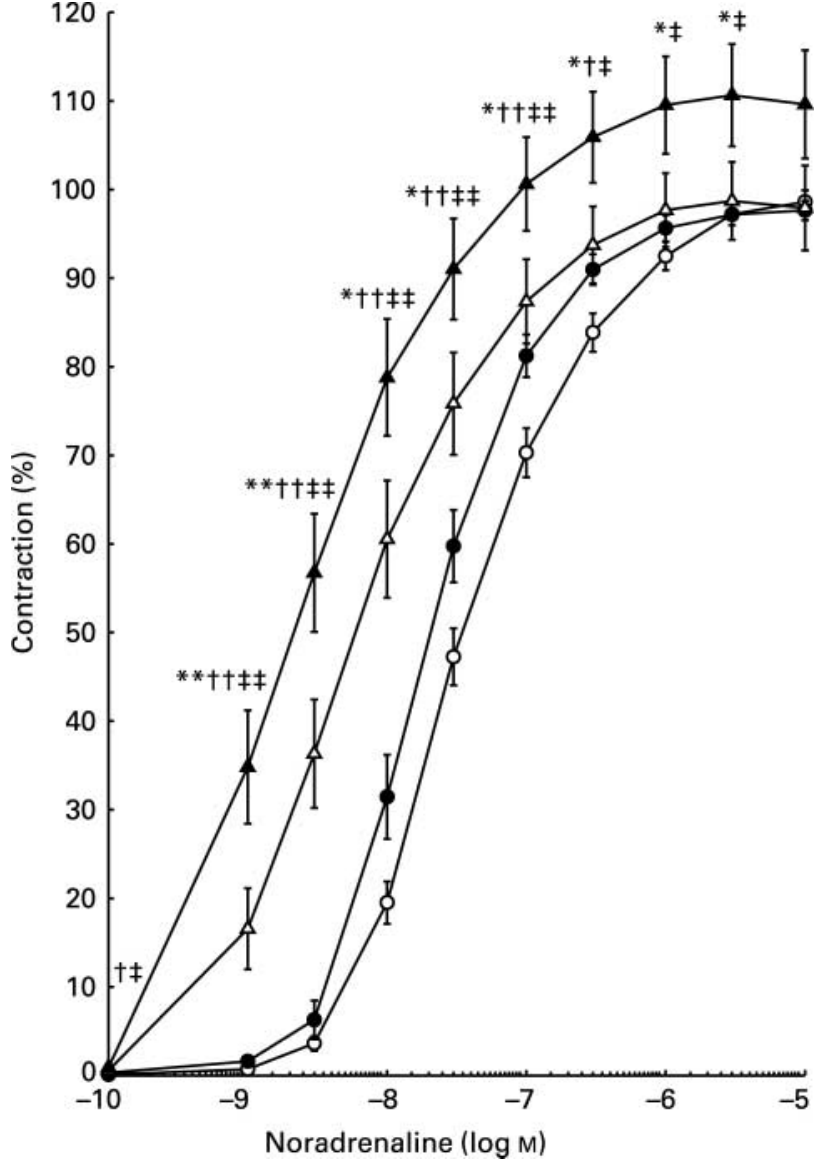

Fig. 3. Effect of a control or methionine (Met)-supplemented diet on the action of noradrenaline on isolated aortic rings from SpragueDawley (SD) and deoxycorticosterone acetate (DOCA)-salt hypertensive rats. O, SD control; $\bullet$, SD-Met; $\triangle$, DOCA control; $\boldsymbol{\Delta}$, DOCA-Met. For details of diets and procedures, see p. 858. Values are means with their standard errors shown by vertical bars for twelve rats per group. Mean values for DOCA-Met group were significantly different from those of the DOCA control group: ${ }^{*} P<0.05$, ${ }^{*} P<0.01$. Mean values for DOCA control group were significantly different from those of the SD control group: $+P<0.05,+\uparrow P<0.01$. Mean values for DOCA-Met group were significantly different from those of the SD-Met group: $\ddagger P<0.05, \ddagger \ddagger P<0.01$.

High concentrations of $\mathrm{Ca}^{2+}\left(>3 \times 10^{-4} \mathrm{M}\right)$ induced a greater constriction of aortic rings from SD rats fed with a Met-supplemented diet than those of SD rats on the control diet. A similar diet-effect was observed in the hypertensive DOCA rats for the highest concentration of $\mathrm{Ca}^{2+}\left(10^{-2} \mathrm{M}\right)$ (Fig. 4).

Aortic reactivity to acetylcholine, bradykinin and sodium nitroprusside

Acetylcholine evoked a significantly less effective relaxation of aortic rings from hypertensive DOCA rats than those from normotensive SD control rats. The Met-supplemented diet significantly decreased the acetylcholineinduced relaxation of aortic rings from the normotensive SD rats, whereas a slight reduction was only observed in the DOCA groups (Fig. 5).

Bradykinin elicited lower relaxation of aortic rings from hypertensive DOCA rats than those from normotensive SD 


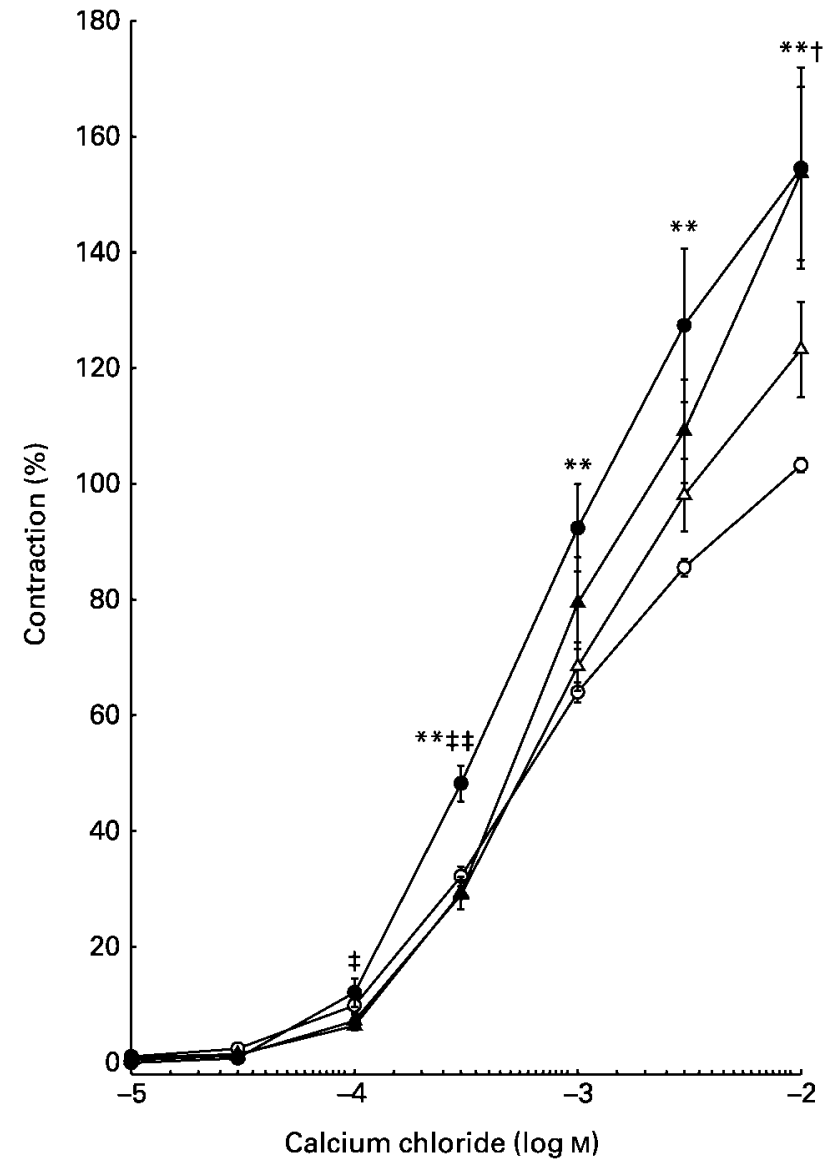

Fig. 4. Effect of a control or methionine (Met)-supplemented diet on the action of calcium chloride on isolated aortic rings from Sprague-Dawley (SD) and deoxycorticosterone acetate (DOCA)-salt hypertensive rats. $\bigcirc$, SD control; $\bullet$, SD-Met; $\triangle$, DOCA control; $\boldsymbol{\Lambda}$, DOCA-Met. For details of diets and procedures, see p. 858. Values are means with their standard errors shown by vertical bars for twelve rats per group. Mean values for SD-Met group were significantly different from those of the SD control group: ${ }^{\star \star} P<0.01$. Mean value for DOCA-Met group was significantly different from that of the DOCA control group: $† P<0.05$. Mean values for DOCAMet group were significantly different from those of the SD-Met group: $\ddagger P<0.05$, $\ddagger P<0.01$.

rats. In normotensive SD rats, the Met-rich diet was associated with an important decrease of the bradykinin-induced relaxation, which became similar to that of DOCA rats. Conversely, aortic rings from DOCA rats fed the Met-supplemented diet were more sensitive to bradykinin than those from control diet DOCA rats (Fig. 6).

Aortic rings from SD rats were significantly $(P<0 \cdot 01)$ more sensitive to sodium nitroprusside than those from DOCA rats. In both normotensive and hypertensive rats the Met-supplemented diet was associated with a slight decrease of responses to the greater sodium nitroprusside concentrations $\left(10^{-7}-3 \times 10^{-6} \mathrm{M}\right)$ (Fig. 7).

\section{Discussion}

In the present study of a model of secondary hypertension, we showed that while a Met-enriched diet induced a 2.7-fold increase of plasma Hcy and a slight increase of the systolic BP in normotensive SD rats, a $7 \cdot 1$-fold increase

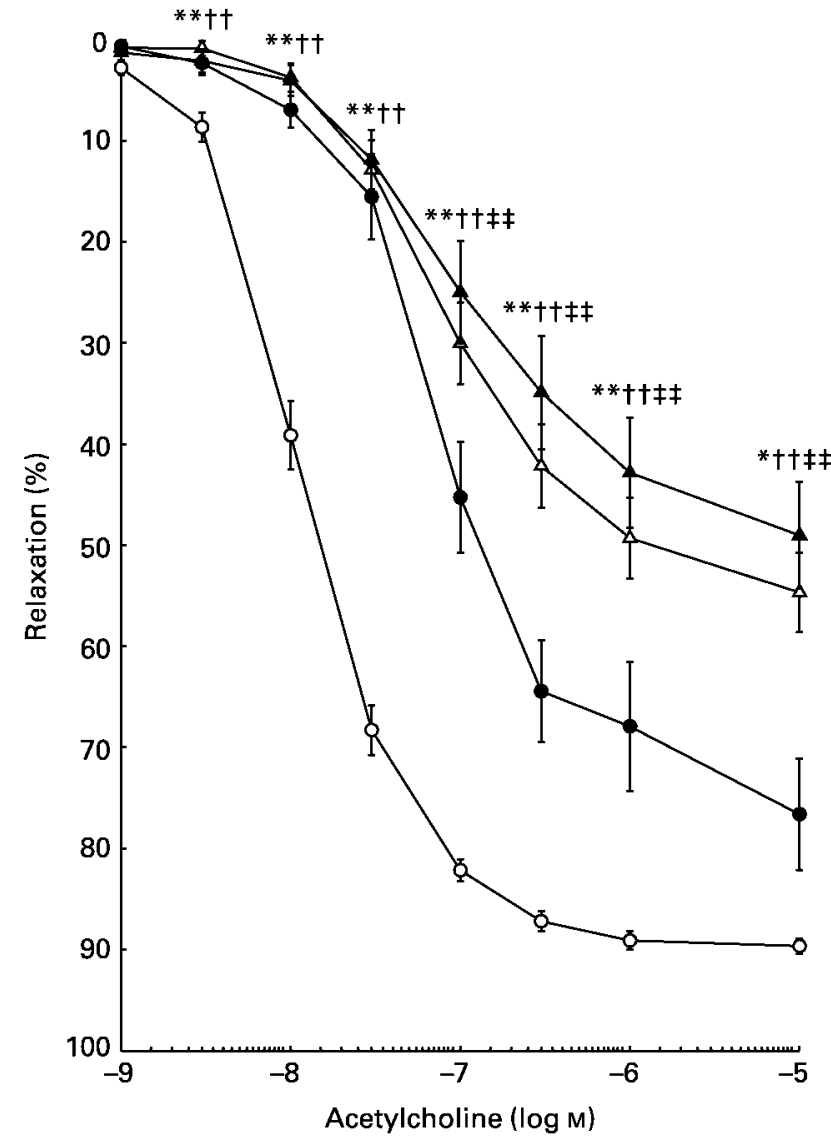

Fig. 5. Effect of a control or methionine (Met)-supplemented diet on the relaxation in response to acetylcholine of isolated precontracted aortic rings from Sprague-Dawley (SD) and deoxycorticosterone acetate (DOCA)-salt hypertensive rats. $\bigcirc, \mathrm{SD}$ control; $\bullet$, SD-Met; $\Delta$, DOCA control; $\boldsymbol{\Lambda}$, DOCA-Met. For details of diets and procedures, see p. 858. Values are means with their standard errors shown by vertical bars for twelve rats per group. Mean values for SD-Met group were significantly different from those of the SD control group: ${ }^{\star} P<0.05,{ }^{\star \star} P<0.01$. Mean values for DOCA control group were significantly different from those of the SD control group: $\dagger+P<0.01$. Mean values for DOCA-Met group were significantly different from those of the SD-Met group: $\ddagger \ddagger P<0 \cdot 01$.

of plasma Hcy was associated with a reduced development of hypertension in DOCA rats. These results agree with a previous study of a model of primary hypertension that showed that the same Met-supplemented diet, while inducing a 3-fold increase of plasma Hcy, increased the systolic $\mathrm{BP}$ in normotensive Wistar-Kyoto rats but decreased systolic BP in SHR (Robin et al. 2003). The Met-enriched diet therefore attenuated the development of hypertension in two models with different hypertensive aetiologies and pathophysiological mechanisms. In normotensive WistarKyoto or SD rats fed the Met-enriched diet, the increase in the systolic BP associated with an increase of plasma Hcy agrees with the biological mechanisms that support a relationship between HHcy and vascular disease. However, the discrepancies between the effect of a similar 3 -fold elevation of Hcy on systolic BP in Wistar-Kyoto and SHR (Robin et al. 2003) and in different gender of SHR (Yen \& Lau, 2002) lead us to suggest that the positive association that has sometimes been reported between plasma Hcy and BP is unlikely to be causal. 


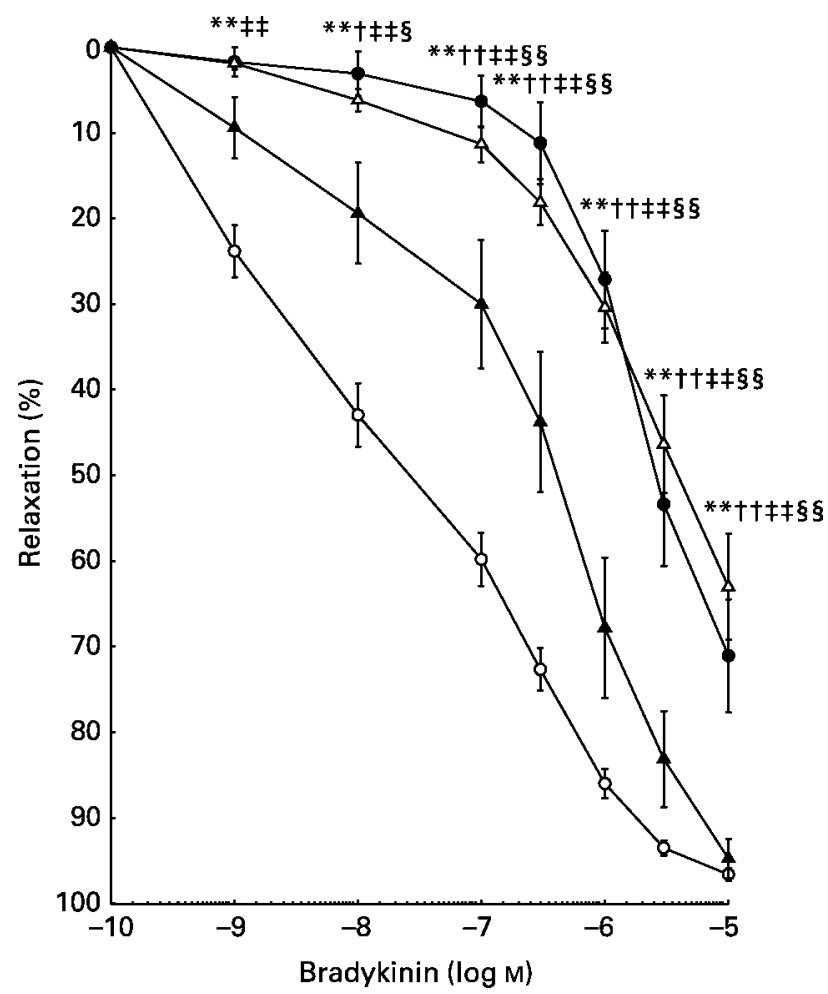

Fig. 6. Effect of a control or methionine (Met)-supplemented diet on the relaxation in response to bradykinin of isolated precontracted aortic rings from Sprague-Dawley (SD) and deoxycorticosterone acetate (DOCA)-salt hypertensive rats. O, SD control; $\bullet$, SD-Met; $\triangle$, DOCA control; $\mathbf{\Lambda}$, DOCA-Met. For details of diets and procedures, see p. 858. Values are means with their standard errors shown by vertical bars for twelve rats per group. Mean values for SD-Met group were significantly different from those of the SD control group: ${ }^{\star \star} P<0 \cdot 01$. Mean values for DOCA-Met group were significantly different from those of the DOCA control group: $\dagger P<0.05$, $\dagger \dagger P<0.01$. Mean values for DOCA control group were significantly different from those of the SD control group: $\ddagger \ddagger P<0.01$. Mean values for DOCA-Met group were significantly different from those of the SD-Met group: $\S P<0.05, \S \S P<0.01$.

This hypothesis is confirmed by the results shown here, since the slower increase in systolic BP of DOCA rats fed the Met-supplemented diet was associated with a higher increase of the plasma Hcy $(\times 7 \cdot 1)$ than in normotensive SD rats $(\times 2.7)$ for which an increase in systolic $\mathrm{BP}$ was observed.

Since the effect of the Met-enriched diet on the systolic BP was not related to plasma Hcy level, these results raise the question of the implication of another metabolite of the Met-Hcy pathway. Hcy results from the transformation of Met into $S$-adenosyl-methionine, which is demethylated into $S$-adenosylhomocysteine and finally converted into Hcy by $S$-adenosylhomocysteine hydrolase. Hcy is either remethylated to Met in the presence of the cofactors betaine and folate or condensed with serine into cystathionine, which is converted to cysteine leading to taurine and GSH synthesis. As expected, the Met supplementation induced a greater degree of HHcy in the DOCA rats than in the SHR; this could be related to an increased expression and activity of the $S$-adenosylhomocysteine hydrolase by DOCA, as reported for aldosterone (Stockand et al. 2001). An alteration of the catabolism of Hcy via

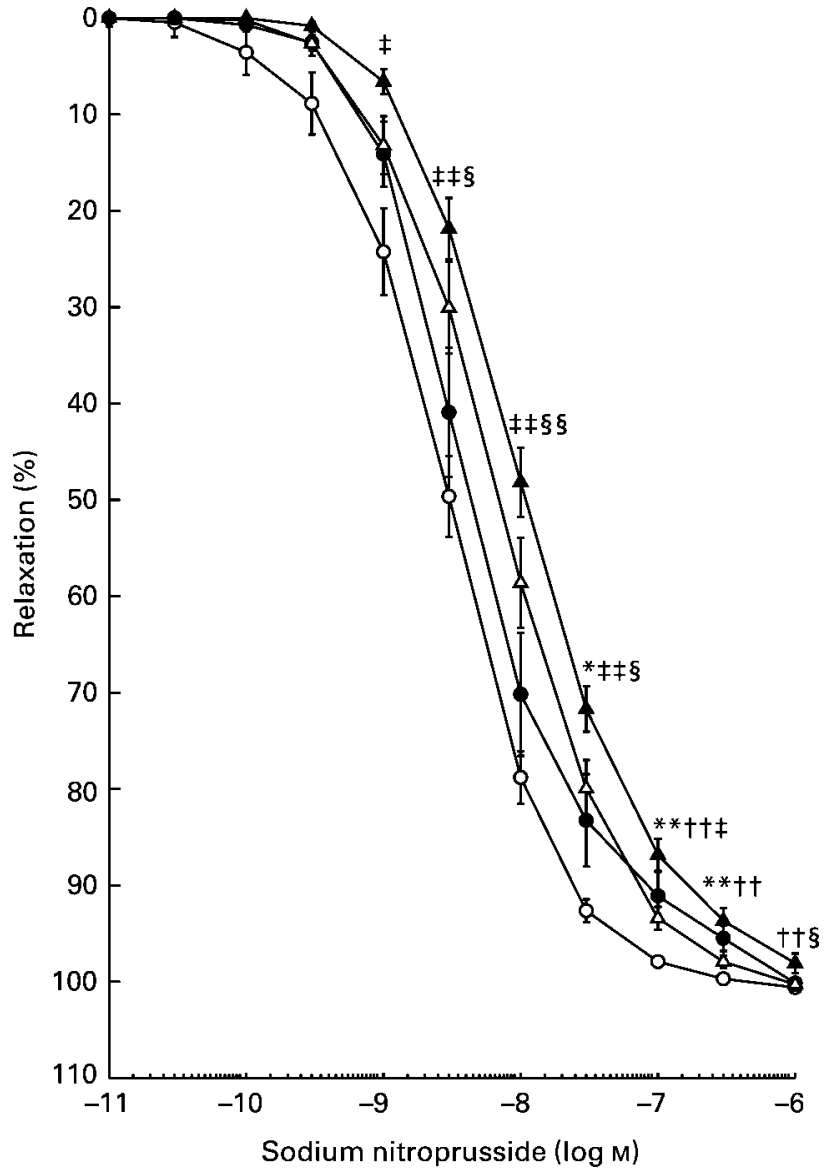

Fig. 7. Effect of a control or methionine (Met)-supplemented diet on the relaxation in response to sodium nitroprusside of isolated precontracted aortic rings from Sprague-Dawley (SD) and deoxycorticosterone acetate (DOCA)-salt hypertensive rats. $O, S D$ control; -, SD-Met; $\triangle$, DOCA control; $\boldsymbol{\Lambda}$, DOCA-Met. For details of diets and procedures, see p. 858 . Values are means with their standard errors shown by vertical bars for twelve rats per group. Mean values for SD-Met group were significantly different from those of the SD control group: ${ }^{*} P<0.05$, ${ }^{\star} P<0.01$. Mean values for DOCAMet group were significantly different from those of the DOCA control group: $\dagger+P<0.01$. Mean values for DOCA control group were significantly different from those of the SD control group: $\ddagger P<0.05$, $\ddagger \ddagger P<0.01$. Mean values for DOCA-Met group were significantly different from those of the SD-Met group: $\S P<0.05, \S \S P<0.01$.

methionine synthase cofactors and cystathionine synthase could also occur. This increased HHcy was not associated with a greater effect on the systolic BP of the DOCA rats than SHR (Robin et al. 2003). The opposite effects of the Met-enriched diet on cysteinaemia and methioninaemia, while inducing a similar systolic BP decrease in the two hypertension models, suggest that these compounds are not related to the modification of systolic BP. The hypothesis that an increase of the GSH levels may be implicated in the lowered systolic BP observed in SHR fed the Metrich diet (Robin et al. 2003) is not verified in the DOCA-salt model, since a decrease of the glutathionaemia in the DOCA-Met rats was associated with a similar BP decrease. Among other metabolites that can be increased when Met is in excess, taurine is effective in inhibiting the development of hypertension in DOCA-salt rats and also in other models of hypertension (for review, see 
Militante \& Lombardini, 2002). It could be suggested that the high Met-diet induced a greater increase in taurine concentration in hypertensive rats than in normotensive rats and that this would result in the hypotensive effect. However, this would assume that the effect of taurine in normotensive rats was not important enough to mask the hypertensive action of Hcy. Clearly, further investigations are required to determine whether the effect of the Metsupplemented diet may be related to taurine.

Previous morphological and functional studies showed that HHcy induced vascular alterations (Matthias et al. 1996; Ungvari et al. 1999; Yen \& Lau 2002; Robin et al. 2003); thus, we further investigated the contractile response and relaxant properties of isolated aortic rings from the four experimental groups. The enhanced responsiveness to vasoconstrictors, impaired endothelial function and decreased responsiveness to NO observed in SD rats fed the Metenriched diet were in accordance with previous results in Wistar-Kyoto rats, in which these alterations were less pronounced (Robin et al. 2003). This was consistent with the observed increase in systolic BP in the normotensive rats fed the Met-enriched diet. In the hypertensive DOCA rats, however, the systolic $\mathrm{BP}$ reduction effect of the Met-supplemented diet was not in agreement with the increased vasoconstrictive response to noradrenaline and the decreased relaxation to acetylcholine and NO donor. However, the increased responsiveness to bradykinin was in accordance with the systolic BP-lowering effect of the Met-supplementation. Discrepancies between the acetylcholine and bradykinin responses in the aortic rings of the DOCA-Met rats suggests that the bradykinin-induced vasodilatation was independent of $\mathrm{NO}$ and mediated by endothelium-derived hyperpolarizing factor. In isolated DOCA rat hearts, Millette et al. (2000) reported that the combination of NO synthase and cyclo-oxygenase inhibition unmasked an involvement of endothelium-derived hyperpolarizing factor in the coronary artery dilation due to bradykinin. This suggested that endothelial NO and prostaglandin $\mathrm{I}_{2}$, although unable to induce relaxation themselves, could inhibit endothelium-derived hyperpolarizing factor production in DOCA-salt rats. Therefore, in this model of DOCA-salt hypertension, the Met-enriched diet could have unmasked and/or increased the involvement of endothelium-derived hyperpolarizing factor in aortic dilation in response to bradykinin. However, whether these vascular alterations were directly connected to the change of BP is unknown. The fact that the Met-supplemented diet had different effects upon the isolated vessels, but the same effect on the development of hypertension in the two models, suggests the involvement of other mechanisms. As in SHR (Robin et al. 2003), systolic BP was not related to body weight and renal function seemed to be unaffected by the diet, since kidney weight, urinary output, urinary and plasma $\mathrm{Na}$ concentrations were not modified by the Met-enriched diet. This suggests that other BP control systems are implicated in the effects of Met supplementation.

In conclusion, our present results, which show that a Met-enriched diet was associated with an increase in systolic BP in normotensive SD rats and with a lesser development in DOCA-salt hypertension rats, are in accordance with a previous study using the SHR genetic model of hypertension (Robin et al. 2003). The differential vasopressive effect of the Met-rich diet and particularly the reduction of the development of the DOCA-salt hypertension, which was associated with a higher HHcy in DOCA than in SD rats, suggest more complex interactions between $\mathrm{HHcy}$ and BP than the positive association described before. The Met-enriched diet cannot only be considered as a HHcy model, since other metabolites and mechanisms seemed to be implicated in these complex interactions. The differential vascular effect of the Met-supplemented diet associated with a similar reduction of the development of hypertension in the two models of hypertension suggests the involvement of other BP control mechanisms. Further investigations are needed to understand the paradoxical effect of a Met-rich diet on systolic BP.

\section{References}

Charpiot P, Bescond A, Augier T, Chareyre C, Fraterno M, Rolland PH \& Garcon D (1998) Hyperhomocysteinemia induces elastolysis in minipig arteries: structural consequences, arterial site specificity and effect of captopril-hydrochlorothiazide. Matrix Biol 17, 559-574.

Fiorina P, Lanfredini M, Montanari A, Peca MG, Veronelli A, Mello A, Astorri E \& Craveri A (1998) Plasma homocysteine and folate are related to arterial blood pressure in type 2 diabetes mellitus. Am J Hypertens 11, 1100-1107.

Hanratty CG, McGrath LT, McAuley DF, Young IS \& Johnston GD (2001) The effects of oral methionine and homocysteine on endothelial function. Heart 85, 326-330.

Kanani PM, Sinkey CA, Browning RL, Allaman M, Knapp HR \& Haynes WG (1999) Role of oxidant stress in endothelial dysfunction produced by experimental hyperhomocyst(e)inemia in humans. Circulation 100, 1161-1168.

McCully KS (1969) Vascular pathology of homocysteinemia: implications for the pathogenesis of arteriosclerosis. Am J Pathol 56, 111-128.

Majors A, Ehrhart LA \& Pezacka EH (1997) Homocysteine as a risk factor for vascular disease enhanced collagen production and accumulation by smooth muscle cells. Arterioscler Thromb Vasc Biol 17, 2074-2081.

Mansoor MA, Bergmark C, Haswell SJ, Savage IF, Evans PH, Berge RK, Svardal AM \& Kristensen O (2000) Correlation between plasma total homocysteine and copper in patients with peripheral vascular disease. Clin Chem 46, 385-391.

Matthias D, Becker CH, Riezler R \& Kindling PH (1996) Homocysteine induced arterosclerosis-like alterations of the aorta in normotensive and hypertensive rats following application of high doses of methionine. Atherosclerosis 122, 201-216.

Militante JD \& Lombardini JB (2002) Treatment of hypertension with oral taurine: experimental and clinical studies. Amino Acids 23, 381-393.

Millette E, de Champlain J \& Lamontagne D (2000) Altered coronary dilatation in deoxycorticosterone acetate-salt hypertension. J Hypertens 18, 1783-1793.

Nakata Y, Katsuya T, Takami S, Sato N, Fu Y, Ishikawa K, Takiuchi S, Rakugi H, Miki T, Higaki J \& Ogihara T (1998) Methylenetetrahydrofolate reductase gene polymorphism: relation to blood pressure and cerebrovascular disease. Am J Hypertens 11, 1019-1023.

Pinto Y, Buikema H, van Gilst WH, Scholtens E, van Geel PP, de Graeff PA, Wagner J \& Paul M (1997) Cardiovascular 
end-organ damage in Ren-2 transgenic rats compared to spontaneously hypertensive rats. J Mol Med 75, 371-377.

Robin S, Maupoil V, Groubatch F, Laurant P, Jacqueson A \& Berthelot A (2003) Effect of a methionine-supplemented diet on the blood pressure of Wistar-Kyoto and spontaneously hypertensive rats. $\mathrm{Br} J$ Nutr 89, 539-548.

Rolland PH, Friggi A, Barlatier A, Piquet P, Latrille V, Faye MM, Guillou J, Charpiot P, Bodard H, Ghiringhelli O, et al. (1995) Hyperhomocysteinemia-induced vascular damage in the minipig. Circulation 91, 1161-1174.

Stockand JD, Zeltwanger S, Bao HF, Becchetti A, Worrell RT $\&$ Eaton DC (2001) $S$-adenosyl-L-homocysteine hydrolase is necessary for aldosterone-induced activity of epithelial $\mathrm{Na}(+)$ channels. Am J Physiol Cell Physiol 281, C773-C785.

Sutton-Tyrrell K, Bostom A, Selhub J \& Zeigler-Johnson C
(1997) High homocysteine levels are independently related to isolated systolic hypertension in older adults. Circulation 96, $1745-1749$.

Taylor LM Jr, Moneta GL, Sexton GJ, Schuff RA \& Porter JM (1999) Prospective blinded study of the relationship between plasma homocysteine and progression of symptomatic peripheral arterial disease. J Vasc Surg 29, 8-19.

Ungvari Z, Pacher P, Rischak K, Szollar L \& Koller A (1999) Dysfunction of nitric oxide mediation in isolated rat arterioles with methionine diet-induced hyperhomocysteinemia. Arterioscler Thromb Vasc Biol 19, 1899-1904.

Yen CH \& Lau YT (2002) Vascular responses in male and female hypertensive rats with hyperhomocysteinemia. Hypertension 40, 322-328. 